\title{
Editorial: Collection on Two-dimensional Materials and Devices
}

The field of nanotechnology appears to be leaping among dimensions. It may have taken off with the extraordinary self-assembly of sub-nanometer zero-dimensional (0D) $\mathrm{C}_{60}$ 'buckyball' molecules and luminescent quantum dots in bulk quantities. Next came one-dimensional (1D) carbon nanotubes with a similar-sized diameter. Before returning to threedimensional (3D) systems, the evolution eventually reached graphene as a prototypical two-dimensional (2D) system, with other 2D materials soon following suit.

Many will agree that the field of Two-dimensional Materials and Devices is now one of the hottest fields in Physics, spanning from new materials to diverse new applications. I was pleased to hear that the Editors of Physical Review Applied and Physical Review Materials shared this opinion and were eager to publicize their support for this evolving field. The idea to highlight their commitment in a collection of selected invited articles on this topic has evolved over time. When I was invited to join this effort as Guest Editor, I agreed with pleasure.

To begin with, the Editors contributed suggestions regarding authors to invite. One important inspiration was the list of invited speakers at the 2016 and 2018 Gordon Research Conferences on "2D Electronics beyond Graphene". Our intention was to represent the uniqueness of the field by inviting contributions from leaders in the field. We ended up with a list of about 50 potential contributors, experimentalists and theorists, covering the entire field of Physics in 2D and its most intriguing aspects. Invitation letters were sent out in late 2019. We were pleased that most invitees committed to contribute an article to this collection.

A typical collection of articles may be viewed as a hybrid between the rigid format of an edited book and a (usually larger) set of articles published in a topical journal. The article collection on Two-dimensional Materials and Devices we introduce here goes one step further, as it combines contributions accepted in two different topical journals with different objectives, namely Physical Review Applied and Physical Review Materials. The Editors' intention is to present around 20-25 quality contributions representing the field of 2D Physics, which should be of primary interest to readers of these journals. Besides being part of this collection, about half of the contributions are expected to be accommodated in either of these two journals. Our plan is to present a 'snap shot' of the field over the course of 2020, bracketed by opening and closing editorials. We have encouraged the authors, who are leaders in the field, to not only report their interesting results, but also to provide an outlook that would be useful as guidance to researchers in the field.

As mentioned above, it is the intention of Physical Review Applied and Physical Review Materials to complete this collection by the end of 2020. I thank the many contributors to this collection for their support.

Published 13 March 2020

David Tománek

Guest Editor

Collection on "Two-dimensional Materials and

Devices"

DOI: 10.1103/PhysRevApplied.13.030001 